\title{
ANALISYS OF ACCESS POINTS WITH THE QUEUE MODEL FOR BIOMETRIC ACCESS CONTROL IN LARGE HEADCOUNT PLANTS
}

\author{
Csaba OTTI \\ University of Óbuda, Budapest, Hungary \\ otti.csaba@bgk.uni-obuda.hu \\ László HANKA \\ University of Óbuda, Budapest, Hungary \\ hanka.laszlo@bgk.uni-obuda.hu
}

\begin{abstract}
The scaling of access control systems is usually done with respect to the life protection requirements regarding escape routes. At large headcount areas, the need for biometric identification arises from the security and business needs. Biometric systems can be characterized by probability variables, which can significantly affect the access process. Mathematically, access control is a discrete state space, stochastic process without memory, that can be described by a queue model. This study demonstrates the process model of access control systems and describes the mathematical model that allows for accurate planning and can ensure a successful introduction for access control systems.
\end{abstract}

KEYWORDS: access control system, biometry, queue model

\section{Introduction}

Waiting queues are found in every aspect of life. Waiting is a natural part of life - however unexpected or unreasonable queues can be a source of major discomfort for users. Parallelly to this, one of the most important tasks in creating a secure environment is to authenticate users for physical access into protected facilities and areas (Berek, 2014, pp. 19-24). Throughput calculations generally do not require serious mathematical modelling or designing problems usually arise when either the access procedure is long due to the security level of the facility (metal detector gates, bag checking) or a large number of people arrive within a short timeframe. An increase in security level means a longer authentication time - which translates into an increased waiting time. The task of security experts is to find the optimal solution. Queue and mass serving models can serve as an adequate base to analyse access procedures and approximate their behavior thus making them plannable (Hillier \& Lieberman, 2014). The purpose of this study is to create a MATAB program with which the properties of various scenarios can be examined, and also to analyse the results through a few examples. The study consists of the following parts: the 2. chapter describes the access process and its states. The 3 . chapter introduces the queue model, and based on these, the 4. chapter develops the model of the access process. The 5. chapter details the mathematical model of the access process and demonstrates this through several calculation examples and the 6. chapter will summarize the study. 


\section{The Access Procedure}

The access control system according to Bunyitai is a: "Complex electromechanics-IT system, that - with the help of installed checkpoints - enables granting or denying personnel and vehicle access based on location, time and direction, while providing logging and tracking” (Bunyitai, 2011, p. 18). The task of an access control system is to: "Identify the person, determine the access right, document the event and control traffic" (Bunyitai, 2011). The general elements of an access control system are readers: identifies the user arriving to the checkpoint. It can be password, card or biometry based - or a combination of them, controllers: they determine whether a user is eligible to access based on the code identified by the readers, APAS: The physical restrictive and mechanical devices controlled by the system and sensors that provide feedback. The controlled devices can be magnetic locks, holding magnets, turnstiles, turning crosses, revolving doors, automatic doors, etc. Sensors can be, or example infra gates, opening detectors or movement detectors. Finally, supervision software: This application serves as a control and display interface to the system settings, logs and handles the incoming signals from the hardware elements.

\section{States of the Access Process}

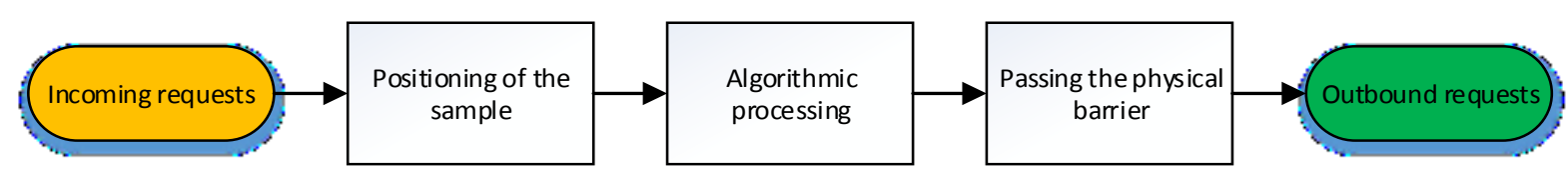

Figure no. 1: The states of the access process

(Source: Otti, 2015)

The states of the access process can be seen in the Figure no. 1. The properties of the individual stages are as such incoming requests: the employee or user arrives to the checkpoint and stands into the queue. Positioning the sample, when the user prepares for identification and presents their biometric sample to the sensor in order to gain access. An analogy to this for cardbased access control is to touch the card to the reader. Algorithmic processing when the reader processes the sample and provides a successful or unsuccessful feedback signal. This step is only applicable for biometric systems, and this is the point where the probability nature of biometrics manifests - as it is never $100 \%$ that a person can pass through the access point at the first try. The other consequence of this property - that also carries a security risk is that an ineligible person gaining access can also not be ruled out by $100 \%$. This probability factor does not exist with card and PIN based systems. Passing the physical barrier, after a successful identification, the controller will signal the physical barrier to grant access. Outbound requests, when the user leaves the checkpoint. In an ideal environment, eligible people can always pass through the checkpoint, while attackers are always denied access, and as such, one must know the points where the system, in reality, may work differently. Incoming requests may face a queue. Positioning the sample can be unsuccessful: for example, the user places their finger on the sensor improperly, or maybe grows a beard and facial recognition systems will not recognize them, or drops the card etc. Algorithmic processing returns a bad result and requires a new attempt. The physical barrier does not work 
properly, the door jams, the turnstile stops turning or the user uses it improperly - for example, tries to pass through too fast, jamming the barrier which will warrant another attempt.

\section{Queue Model}

Queues appear in various aspects of life, where access to a distributed resource is being served. Any system, where the customer is served with a limited resource, can be considered a queue system (Kleinrock, 1975). Example for such a system is a line in order to get ice-cream, a queue in the bank, the landing and maintenance queue for aircraft, data processing in a computer processor or even the students waiting for an exam. According to Pokoradi "By queue, service systems, we mean a system where consumers arrive at random, the various requests wait for service, then depart" (Pokorádi, 2008, pp. 173-175). Queue systems are also called Mass Service Systems. Queuing problems can be estimated, analysed and evaluated by analytic modelling or simulations. The analytic methods can be used with simpler queues, where the equations of the model can be easily obtained by simplifying certain aspects of the real process. In reality, however, it is very hard to describe such a system, because not all factors can be considered, or the equations obtained have a non-polinomial algorithmic processing time (Lovász, 2009, pp. 42-43). In these cases, an efficient examination method is simulation. That is done by simulating the operation of the system with a large element number and we derive conclusions from the results (Szeidl, 2009, p. 78). These systems have the following in common: the architecture of the system, incoming requests, waiting queues, servers, services, outbound requests.

Stochastic Process: It is not an uncommon issue in the technical sciences that the evolution of basic quantities required for analysis depends entirely on chance. These quantities typically describe the temporal and/or spatial changes of the analyzed factor. In this case, we can interpret quantities as a collective of probability variables belonging to the parameter. If the parameter set becomes a subset of the positive half-line, then can be considered as a time parameter or, in short, time. The set of real numbers is orderly, and as such the past and the future of the process can be interpreted. If we consider fixed value as present, then we can interpret as the future of the process, while is the past of the process (Pap \& Szücs, 2014, pp. 3-5).

Markov Process: We can describe a stochastic process as a Markov process, if the future states of the process are only influenced by the past states through the present states - or in other words, the process is without memory. For example, if five people stand at a turnstile access point, it doesn't matter that this state occurred because originally there were six people but one passed through or originally there were only three people and two more arrived. The access process can be considered a continuous, discrete state space Markov process - or in other words, a Markov chain. Every state in the system shows the number of people in queue and being served. The increase of waiting elements in the system is described by $\lambda$ - arrival intensity, the decrease is described by $\mu-$ serving intensity. The base state of the system is that it is empty.

The Kendall Notation System: Kendall has published the general notations required to describe mass service systems in 1953. Based on this, the types of queue systems can be described if we know the incoming distribution, the properties of the queue and the service mechanism (Kendall, 1953, pp. 338-340). The purposes of this study are best served by the model of the book 'Basis of queuing theory' by 
Sztrik (2011). The notations used to describe queuing systems are:

$$
A / B / s / K / n / E
$$

where:

$-\mathrm{A}=$ the distribution function of incoming request times.

$-\mathrm{B}=$ the distribution function of service times.

$-\mathrm{s}=$ the number of servers.

- $\mathrm{K}=$ the capacity of the system, or, in other words, the maximum number of requests that can stand in line.

$-\mathrm{n}=$ the amount of request sources.

$-E=$ the basis of service.

The distribution functions (A, B) can be deterministic (D), exponential (M) or general $(\mathrm{G})$. The capacity of the system $(\mathrm{K})$ and the source of requests can be (n) finite or infinite - we generally use the latter. The basis of the service can be (E) FIFO (First In First Out), LIFO (Last In First Out), random or based on priority.

\section{Terminology and Notation:}

The state of the system $=$ the number of waiting elements in the queue.

- Queue length = the number of waiting elements that are waiting for the service to start.

- $N(t)=$ the number of elements waiting at $t(t \geq 0)$ point in time

- $P_{n}(t)=$ the probability of exactly $n$ elements being present in the system at any given $t$ moment.

$-s=$ the number of parallel servers in the system.

$-\lambda=$ incoming intensity per time unit.

$-\mu=$ service intensity per time unit.

$-\varrho=\frac{\lambda}{s \mu}=$ utilisation factor.

When a system is stable and set in (the queuing models - as this study does so as well - generally examine this state), then:

- $P_{n}=$ is the probability, that exactly $n$ elements are waiting in the system.

- $L=\sum_{n=0}^{\infty} n P_{n}=$ the number of elements waiting in the system.

- $L_{q}=\sum_{n=s}^{\infty}(n-s) P_{n}=$ expectable queue length.
- $\mathrm{W}=$ service time in the system with waiting time accounted for.

- $W_{q}=$ time in the queue.

The following equations provide the connection between the above notations: $L=\lambda \mathrm{W}$, called Little formula (Little, 1961, pp. 383-387), meaning: the average number of requests within the system is equal the incoming intensity times the average time spent in the system, where $L_{q}=\lambda W_{q}$ and $W=W_{q}+\frac{1}{\mu}$.

\section{Model of the Access Process}

Access control systems can be described as a multiple server, parallel service system. To further examine queue systems mathematically, we must put a number of restrictions in place in the conditions. These do not substantially influence the realisticity of the model, but if we must deviate from them, there are simulation methods to account for this (Law, 2015). The conditions will be accounted for using the Kendal notation. The distribution of the incoming requests is a Poisson distribution, the service time distribution is also exponential and the number of servers is $m-$ a finite, natural number. The cardinality of the capacity of the system and the source of requests is infinite, the basis for service is FIFO. Based on this, the model of access control systems is: $M / M / s / \infty / \infty / F I F O$. In these cases, the last three parameters are usually not noted in writing - based on this, a one channel access control system is a $M / M / 1$, a multichannel is a $M / M / s$ model mass service system. With the general sense of safety decreasing in the world, an ever-increasing need for authentic identification of users arises. The only technology that allows for identifying personally unique and preferably unfalsifiable properties is biometric identification. The current systems are by no means invulnerable, however, due to constant development, they fit an ever-growing standard of security and 
convenience (Otti, 2016, pp. 251-253). Classification of biometric technologies: first of all, imaging-based technologies (fingerprint recognition, iris recognition, face recognition, vein recognition, hand geometry recognition, signature recognition), and also not (or not directly) imaging-based technologies (voice recognition, DNS test, behavior-based tests). The Figure no. 1 in chapter States of the access process shows "Positioning of the sample" and "Algorithmic processing" that are affected by biometric recognition. The queue model is modified by the service factor of the biometric devices. Service - in contrast to the traditional identification methods - is a probability variable, mostly affected by the FRR (False Rejection Rate) value of the system. $\xi$ probability variable can be defined thusly: Let $r$ be the number of users that within a given timeframe that are rejected by the system, if the enrolled number of users is n. If so, then $\xi$ by definition has a binomial distribution:

$$
P(\xi=r)=\left(\begin{array}{l}
n \\
r
\end{array}\right) p^{r}(1-p)^{n-r} ; r=0,1,2, \ldots, n
$$

The relative probability stochastically converges on $p$ probability, if the number of observations $n$ is increasing beyond all limits. If we wish to estimate this parameter, then the best method is Maximum Likelihood, that, in our case, is equal to the FRR itself. A more detailed deduction can be found in Hanka's publication (Hanka, 2013). From this definition of FRR - which our measurements confirm - comes that the probable run time for the algorithm is the highest in this case compared to any successful identification, since to establish a false rejection, the entire database has to be checked against the present sample (in $1: \mathrm{N}$ identification, where no preselection exists with PIN or card), and the users must present the sample again, which means another full run of the identification cycle. These two factors increase the time required for a false rejection to around two- or threefold of a normal identification. This also means that the dispersion of service times is greatest for biometric identification, furthermore, FRR directly affects service performance, which is critical for access control and attendance tracking applications (Hanka \& Werner, 2015, pp. 209-216).

The purpose of access control is typically tied to the operation of a physical barrier, however in high-security facilities, security methods beyond this are usually deployed. The Table no. 1 summarizes the typical elements and the service times given by the manufacturer versus the empirically obtained ones.

Table no. 1

The typical elements of an access process

\begin{tabular}{|c|c|c|c|c|}
\hline Name & $\begin{array}{l}\text { Service } \\
\text { time (s) }\end{array}$ & Average (s) & (service/minute) & Notes \\
\hline Card based identification & $1-2$ & 1,5 & 40 & \\
\hline PIN code & $1-4$ & 2,5 & 24 & \\
\hline Biometric identification & $1-9$ & 5 & 12 & $\begin{array}{c}\text { The large dispersion is due } \\
\text { to FRR }\end{array}$ \\
\hline Door & $0-2$ & 1 & 60 & $\begin{array}{l}\text { Magnetic lock, door } \\
\text { holding magnet. }\end{array}$ \\
\hline Turnstile, turning cross, fast gate & $2-3$ & 2,5 & 24 & $\begin{array}{l}\text { 20-30 person/minute } \\
\text { throughput }\end{array}$ \\
\hline Turnstile, one person & $3-10$ & 6,5 & 9,23 & \\
\hline Guest registration & $30-180$ & 105 & 0,57 & $\begin{array}{l}\text { ID card checking, data } \\
\text { recording, issuing the card, } \\
\text { notifying escort. }\end{array}$ \\
\hline Bag x-ray & $30-150$ & 90 & 0,67 & \\
\hline Metal detector gate & $10-30$ & 20 & 3 & \\
\hline Body search & $20-60$ & 40 & 1,5 & \\
\hline
\end{tabular}




\section{Mathematical Model of the System}

The mathematic model was mainly developed based on the work of Hillier and Lieberman: "Introduction to operations research". Beyond this, we also utilized "The basis of queuing theory" from János Sztrik, "Queue Modelling and Simulation" from Fischwick, and "Development and introduction of access gate placement strategy demonstrated through a select number of metro stations" from József Lukács (Hillier \& Lieberman, 2014; Sztrik, 2011; Fishwick \& Hyungwook, 2008; Lukács, 2014). In this article the access control system is considered as a queuing system. Customers are registered users in the system, and the service is the "access" it self. Consequently, the mathematical description of control system is given by the characteristic values of a queuing system. These essential values are the average number of customers in the system, expected queue length, the mean waiting time in the system, including service time, and the expected waiting time in the queue, denoted by $L, L_{q}, W$ and $W_{q}$ respectively. The value of these quantities is the most important question for the employer and for employees as well. These values depend on the mean arrival rate, and mean service rate, denoted by $\lambda$ and $\mu$ respectively. These quantities are by definition the number of arrivals and the number of served customers per unit time respectively. The reciprocal of these values has illustrative meaning, these are mean interarrival time and mean service time respectively. In general, these rates may depend on the number of customers in the system, but it is acceptable, that considering an access control system, the arrival rate and the service rate is independent to the state of the system, in other words, to the number of customers, hence these quantities are constant. The following fundamental question is the number of channels in the system, which is denoted by $s$. Obviously, if the number of registered users is great enough, a singleserver system is not satisfactory, consequently a multiple-server system is necessary. The appropriate number of channels is the fundamental question of this article, and is discussed below. Moreover, since arrivals and services are independent, it is also obvious that the interarrival time distribution and the service time distribution can be given by exponential distribution, so the $M / M / 1$ and the $M / M / s$ model can be applied for the access control system. The state of the system is always given by the probability distribution $P_{n}(t)$, which denotes the probability of the event, that there are $n$ customers in the system at time $t$. This distribution depends on $t$ in general, but if the utilization factor, $\rho=\frac{\lambda}{\mu s}$ is less than 1 , the system can reach the steady state condition, therefore the distribution in this case is independent to time, and expected values can be calculated. $L, L_{q}, W$ and $W_{q}$ are the interested expected values. The relationships between these expected values, and the simplest mathematical formulas at the same time are Little's formulas:

$L=\lambda W ; L_{q}=\lambda W_{q} ; W=W_{q}+\frac{1}{\mu}$. If at

least one of the four quantities are known, every other can be calculated using these equations. The mathematical formulas are much simpler if the calling population, in other words the number of registered users is infinity. But apparently, the size of the population is always finite, therefore the difference between finite and infinite mathematical model must be studied for the first time. Due to Little's formulas, it is enough for example focusing on $L_{q}$. For the single-server system, if the size of the population is infinity, $L_{q}$ can be calculated using the following formulas: 


$$
P_{0}=\left[\sum_{n=0}^{\infty}\left(\frac{\lambda}{\mu}\right)^{n}\right]^{-1}=1-\rho ; \quad P_{n}=P_{0} \rho^{n} ; \quad L_{q}=\sum_{n=1}^{\infty}(n-1) P_{n}=\frac{\lambda^{2}}{\mu(\mu-\lambda)} ; \quad \rho=\frac{\lambda}{\mu}
$$

If the size of the population is finite, let it be $N$, then the difference is that the different and the sum in the definition of $L_{q}$ is a finite sum: probability distribution $P_{n}$ is obviously

$$
P_{0}=\left[\sum_{n=0}^{N}\left(\frac{\lambda}{\mu}\right)^{n}\right]^{-1} ; P_{n}=P_{0} \rho^{n} ; \quad L_{q}=\sum_{n=1}^{N}(n-1) P_{n} .
$$

But these results can be given by much complicated formulas. For the multiple-server system, if the size of the

population is infinity, $L_{q}$ can be calculated using the following formulas:

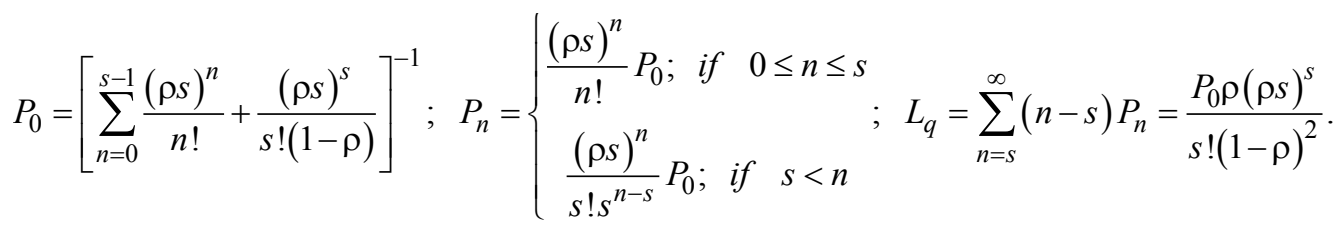

where in this case $\rho=\frac{\lambda}{s \mu}$. If the size of the population is $N$, assuming that $N>s$, the difference is that the second term in the definition of $P_{0}$ must be replaced by the $\operatorname{sum} \frac{(\rho s)^{s}}{s !} \sum_{n=s}^{N} \rho^{n-s}, P_{n}$ can be calculated in the same way if $n \leq N$, and the expected value of queue length is given by the following sum: $L_{q}=\sum_{n=s}^{N}(n-s) P_{n} . \quad$ The outcome of the calculations for the finite case is also much complicated. Comparing finite and infinite model, let's consider a hypothetic situation. Assume for example, that $\lambda=7, \mu=10$ and that the number of registered users is extremely low, for example $N=10$. It can be seen immediately, that difference between models is observable only if $s=1$. If $s \geq 2$ then the curves are practically coincided. Remarkable difference can be observed only if the number of channels is $s=1$. But if the number of registered users is a few hundred, the single-server case is not satisfactory at all. Taking into consideration a situation, when the size of the calling population is 500 , the mean arrival rate and mean service rate for a particular unit time is $\lambda=50$ and $\mu=70$, the corresponding expected values can be seen in Tables no. 2 and no. 3 .

Table no. 2

The expected values of customers in the system in case of infinite and finite model, if $N=500$

\begin{tabular}{|c|c|c|c|c|}
\hline & \multicolumn{2}{|c|}{$L$} & \multicolumn{2}{c|}{$L_{q}$} \\
\hline channels & infinite & finite & infinite & finite \\
\hline 1 & 2.500000000000000 & 2.500000000000001 & 1.785714285714286 & 1.785714285714286 \\
2 & 0.818713450292398 & 0.818713450292398 & 0.104427736006683 & 0.104427736006683 \\
3 & 0.726443355119826 & 0.726443355119826 & 0.012157640834111 & 0.012157640834111 \\
4 & 0.715690500989644 & 0.715690500989644 & 0.001404786703930 & 0.001404786703930 \\
5 & 0.714433200854997 & 0.714433200854997 & 0.000147486569283 & 0.000147486569283 \\
6 & 0.714299566011384 & 0.714299566011384 & 0.000013851725669 & 0.000013851725669 \\
7 & 0.714286880352793 & 0.714286880352793 & 0.000001166067078 & 0.000001166067078 \\
\hline
\end{tabular}


Table no. 3

The expected values of time in case of infinite and finite model, if $N=500$

\begin{tabular}{|c|c|c|c|c|}
\hline & \multicolumn{2}{|c|}{$W$} & \multicolumn{2}{c|}{$W_{q}$} \\
\hline channels & infinite & finite & infinite & finite \\
\hline 1 & 0.050000000000000 & 0.050000000000000 & 0.035714285714286 & 0.035714285714286 \\
2 & 0.016374269005848 & 0.016374269005848 & 0.002088554720134 & 0.002088554720134 \\
3 & 0.014528867102397 & 0.014528867102397 & 0.000243152816682 & 0.000243152816682 \\
4 & 0.014313810019793 & 0.014313810019793 & 0.000028095734079 & 0.000028095734079 \\
5 & 0.014288664017100 & 0.014288664017100 & 0.000002949731386 & 0.000002949731386 \\
6 & 0.014285991320228 & 0.014285991320228 & 0.000000277034513 & 0.000000277034513 \\
7 & 0.014285737607056 & 0.014285737607056 & 0.000000023321342 & 0.000000023321342 \\
\hline
\end{tabular}

Subsequently if the size of calling population is a few hundred and if the finite model is applied for modelling, the obtained data are exactly equal to corresponding data obtained in the infinite model. Therefore, the infinite queuing model can be applied for modelling an access control system in which there are finite number of registered users.
Characteristic values of a queuing system depend on the utilization factor as well. Using formulas for $L_{q}$ and applying Little's formulas, the correspondence can be illustrated. Figure 2 depicts the dependence of $L$ and $W$ on the utilization factor for various numbers of channels using logarithmic $y$-scale, for a particular value of mean arrival rate $(\lambda=10)$.
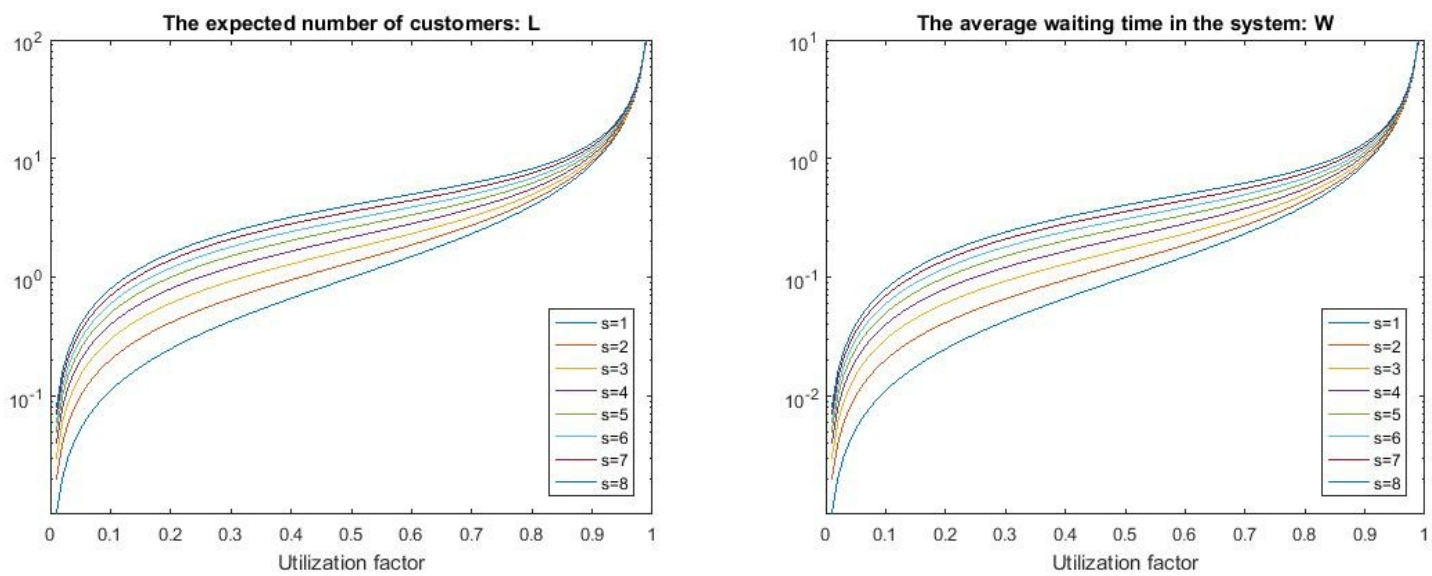

Figure no. 2: The expected number of customers in the system (left) and the average waiting time in the system, including service time (right)

(Source: own edit)

Due to Little's formulas, $\lambda W=L$, hence the relationship between $W$ and $L$ only a constant factor, the shape of curves above are similar. These relationships can be used for planning an access control system. For example, if there is a requirement for the waiting time in the system, the planner can determine for instance the appropriate number of channels. Taking into consideration a real problem, assume that in a particular access control system, the average service time is $13 \mathrm{~s}$. The number of customers is known between 6:00 a.m. and 7.00 a.m. According to observations, the average number of users between 6:00 to $6: 20$ were 185 , between $6: 20$ to $6: 40$ were 275 and between 6:40 to 7:00 were 202 . Therefore, the unit time in this case is $20 \mathrm{~min}$. Since the average service time is $13 \mathrm{~s}$, the mean service rate is $\mu=\frac{1200}{13}=92.3$. Since the system has steady state probability distribution only if the utilization factor is less than 1, taking 
into account the maximal number of arriving customers, the number of channels must satisfy the following inequality:

$$
\rho=\frac{\lambda}{s \mu}=\frac{275}{93.1 \cdot s}<1 \Rightarrow s \geq 3
$$
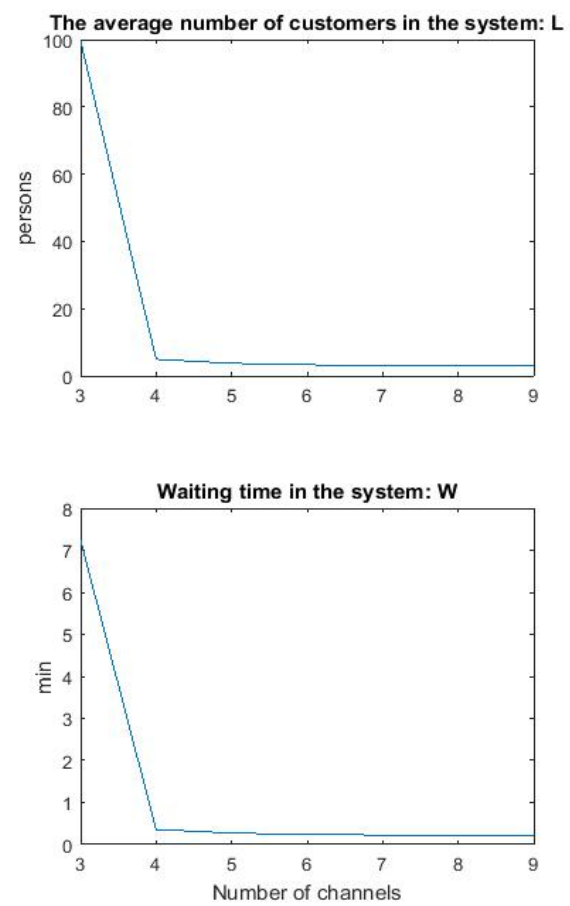

Figure no. 3: Characteristic values for the system given by parameters: $\lambda=275, \mu=92.3, T=20 \mathrm{~min}$.

It can be seen that if the number of channels is 3 , then either the waiting time in the system or the waiting time in the queue is approximately 7 minutes, which is unacceptable for the employees. But if the number of channels is at least 4 , these waiting times are less than 1 minute. Moreover, it is also obvious, that if $s$ is at
Therefore at least 3 channels must be applied in the system. The requirement is that, the average waiting time must be less than 1 minute. Using these data, $L, L_{q}, W$ and $W_{q}$ can be computed if $s$ is at least 3 . The outcome of calculations can be seen on Figure no. 3.
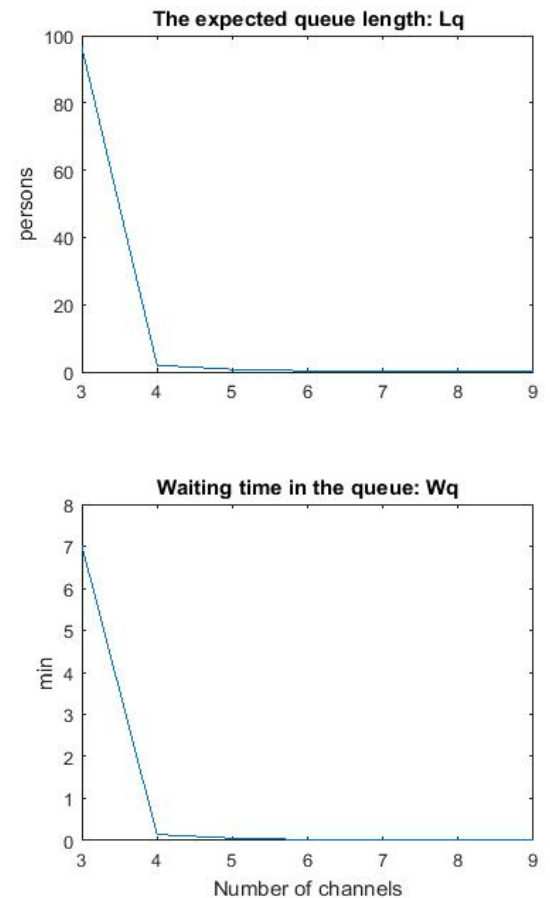

least 4 the time functions and the queue length function are approximately constant functions, consequently $s=4$ the optimal decision. The growing number of channels won't improve the characteristic values, every expected value practically stays the same. Table no. 4 comprises the calculated data for this particular case.

Table no. 4

The characteristic values of the system for various channels if $\lambda=275, \mu=92.3, T=20 \mathrm{~min}$

\begin{tabular}{|c|c|c|c|c|}
\hline number of channels & $L$ (persons) & $L_{q}$ (persons) & $W(\min )$ & $W_{q}(\min )$ \\
\hline 3 & 99.5432 & 96.5638 & 7.2395 & 7.0228 \\
\hline 4 & 4.9559 & 1.9765 & 0.3604 & 0.1437 \\
\hline 5 & 3.7471 & 0.7677 & 0.2725 & 0.0558 \\
\hline 6 & 3.3011 & 0.3217 & 0.2401 & 0.0234 \\
\hline 7 & 3.1072 & 0.1278 & 0.2260 & 0.0093 \\
\hline 8 & 3.0261 & 0.0466 & 0.2201 & 0.0034 \\
\hline 9 & 2.9950 & 0.0155 & 0.2178 & 0.0011 \\
\hline
\end{tabular}


The remaining question can be the following. The actual number of customers is random; therefore, it can be described by a probability distribution. The average number of the customers can be seen in Table no. 4. But these data are "only" expected values of a probability distribution. The question can be the probability of the event, that there are a specified number of customers. Table no. 5 comprises these probabilities and Figure no. 4 illustrates these probabilities graphically.

Table no. 5

The probability of the event that there are $n$ customers in the s-server system

\begin{tabular}{|l|clllllllllll|}
\hline & $\mathrm{n}=0$ & $\mathrm{n}=1$ & $\mathrm{n}=2$ & $\mathrm{n}=3$ & $\mathrm{n}=4$ & $\mathrm{n}=5$ & $\mathrm{n}=6$ & $\mathrm{n}=7$ & $\mathrm{n}=8$ & $\mathrm{n}=9$ & $\mathrm{n}=10$ \\
\hline $\mathrm{s}=3$ & 0.0015 & 0.0046 & 0.0068 & 0.0068 & 0.0067 & 0.0067 & 0.0066 & 0.0066 & 0.0065 & 0.0065 & 0.0065 \\
$\mathrm{~S}=4$ & 0.0389 & 0.1160 & 0.1727 & 0.1716 & 0.1278 & 0.0952 & 0.0709 & 0.0528 & 0.0393 & 0.0293 & 0.0218 \\
$\mathrm{~S}=5$ & 0.0477 & 0.1422 & 0.2119 & 0.2104 & 0.1567 & 0.0934 & 0.0556 & 0.0332 & 0.0198 & 0.0118 & 0.0070 \\
$\mathrm{~s}=6$ & 0.0500 & 0.1490 & 0.2220 & 0.2205 & 0.1642 & 0.0978 & 0.0486 & 0.0241 & 0.0120 & 0.0059 & 0.0030 \\
$\mathrm{~S}=7$ & 0.0506 & 0.1508 & 0.2247 & 0.2231 & 0.1662 & 0.0990 & 0.0492 & 0.0209 & 0.0089 & 0.0038 & 0.0016 \\
$\mathrm{~S}=8$ & 0.0508 & 0.1513 & 0.2253 & 0.2238 & 0.1667 & 0.0993 & 0.0493 & 0.0210 & 0.0078 & 0.0029 & 0.0011 \\
$\mathrm{~s}=9$ & 0.0508 & 0.1514 & 0.2255 & 0.2240 & 0.1668 & 0.0994 & 0.0494 & 0.0210 & 0.0078 & 0.0026 & 0.0009 \\
\hline
\end{tabular}

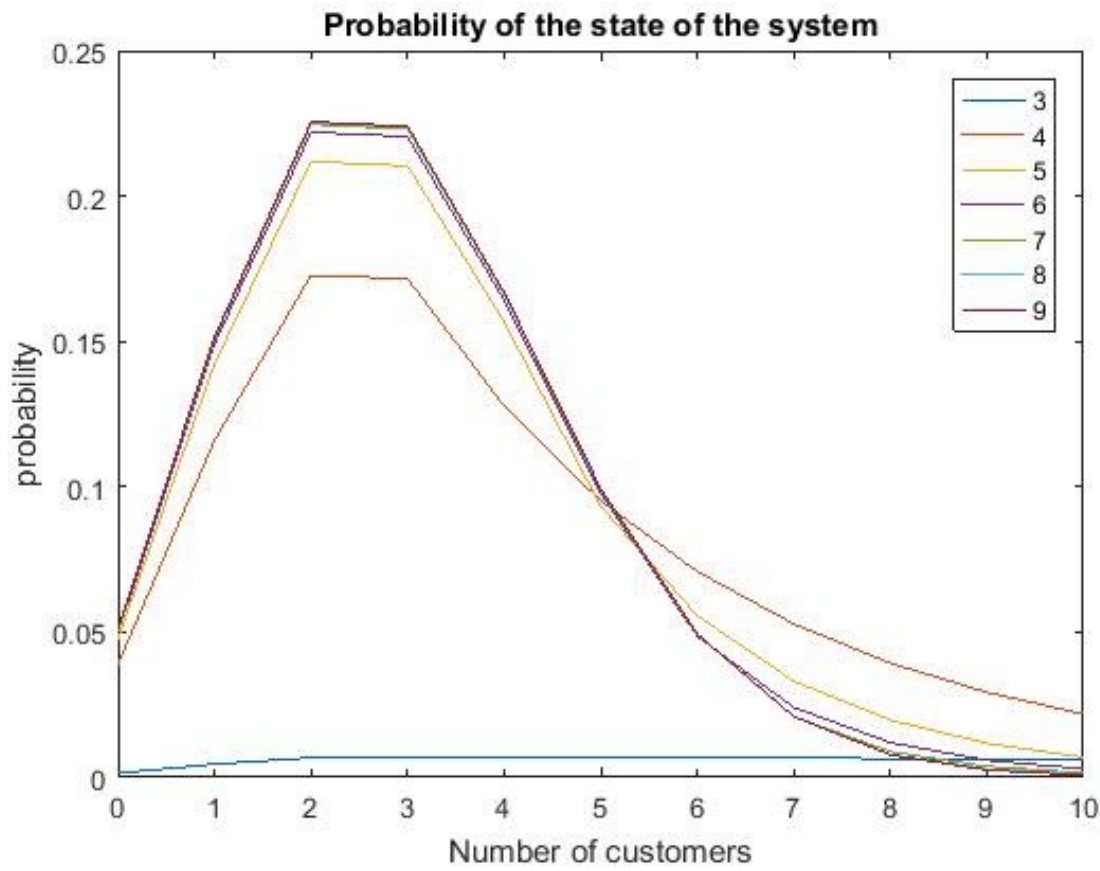

Figure no. 4: The probability of the particular state of the system

\section{Summary}

The study demonstrated a useful method for constructing a stochastic model with a Markov chain for access control systems and new analysis methods based on said method. We can state, based on the results of this study, that this analysis method is suitable for ensuring quality and supporting business decisions through the design phase of biometric or generic access control systems. The goal of the authors is to ensure successful introduction of access control projects for high headcount facilities with respect to business and security requirements and standpoints, to create process- and system analysis methods through mathematical simulation, and - by using case studies - to demonstrate its practical usability. These goals are fulfilled, and in practice, the designing engineers of access control systems are given a new and powerful methodology that is useful in practice. 


\section{REFERENCES}

54/2014. (XII. 5.) BM Decree on the National Fire Protection Regulations (2014). Budapest: National Legislation.

Berek, L. (2014). Security Systems. Budapest: National University of Public Service.

Bunyitai, A. (2011). Location and role of access control systems in asset protection. Budapest: Hadmernok, VI.(4.), 17-25.

Fishwick, P. A., \& Hyungwook, P. (2008). Queue Modeling and Simulation. In Principles of Modeling and Simulation: A Multidisciplinary Approach. New Jersey, USA: John Wiley \& Sons, Inc.

Hanka, L. (2013). Applications for using binomial distribution in functioning identification systems, the application of maximum likelihood principle. Spring Technical Symposium, Budapest, Hungary: University of Obuda.

Hanka, L., \& Werner, G. (2015). Using the Beta-Binomial Distribution for the Analysis of Biometric Identification. 13th International Symposium on Intelligent Systems and Informatics: Proceedings, 209-216, Subotica, Serbia.

Hillier, F. S., \& Lieberman, G. J. (2014). Introduction to Operations Research. New York, USA: McGraw-Hill Higher Education.

Hungarian Standards Institution. (2015). MSZ EN 60839-11-2:2015. Alarm systems and electronic security systems. Part 11-2.: Electronic access control systems. Application Guidelines. Budapest: Hungarian Standards Institution.

Kendall, D. G. (1953). Stochastic processes occurring in the theory of queues and their analysis by the method of imbedded Markov chain. Annals of Mathematical Statistics, Vol. 24, Issue 3, 338-354.

Kleinrock, L. (1975). Queueing Systems Volume 1: Theory. New York: Wiley Interscience.

Law, A. M. (2015). Simulation Modeling and Analysis. 5th Edition. Tucson, Arizona, USA: McGrow-Hill .

Little, J. D. (1961). A proof of the queuing formula: $1=\lambda \mathrm{w}$. Operations Research, Vol. 9(3), pp. 383-387, available at: http://fisherp.scripts.mit.edu/wordpress/wpcontent/uploads/2015/11/ContentServer.pdf

Lovász, L. (2009). Complexity of Algorithms. Budapest: ELTE, Institute of Mathematics.

Lukács, J. (2014). Develop and present an access gate placement strategy through a few selected subway stations. Budapest: BME Budapest University of Technology and Economics.

Otti, C. (2015). Classification of biometric access control systems based on real-time throughput. Proceedings of Fifth International Scientific Videoconference of Scientists and PhD. students or candidates, Bratislava, 63-71.

Otti, C. (2016). Biometric Systems User Pattern Positioning Issues. DOSZ, Spring Wind Conference, 251-260, Budapest.

Pap, G., \& Szücs, G. (2014). Stochastic processes. Szeged, Hungary: STE Institution of Bolyai, Stochastic Department. Kiado.

Pokorádi, L. (2008). Modeling of systems and processes. Debrecen, Hungary: Campus

Szeidl, L. (2009). Mass Service. Budapest, Hungary: University of Obuda, Institute of Informatics.

Sztrik, J. (2011). Basics of queuing theory. Debrecen, Hungary: University of Debrecen. 